\title{
ON A GENERALIZATION OF THE WILSON-GLAISHER THEOREM
}

\section{BY GIOVANNI RICCI}

1. Introduction. J. W. L. Glaisher* has shown that, if $n$ be any number, $p$ any odd prime not exceeding $n, k$ the integral part of the quotient $n / p$, and if $A_{r}$ denote the sum of the products of the first $n-1$ consecutive in tegers taken $r$ together, then

$$
A_{p-1}+k \equiv 0 \quad(\bmod p)
$$

This theorem contains Wilson's theorem as the special case $n=p$, and it has been extended by R. E. Moritz $\dagger$ in the following form. If $n=k p+q, p$ an odd prime, $0 \leqq q<p$, and if ${ }^{m} A_{r}$ denote the sum of the products of any $n-1$ consecutive numbers, $m+1, m+2, \cdots, m+n-1$ taken $r$ together; if $0<q<p$, then ${ }^{m} A_{p-1}+k \equiv 0,(\bmod p)$. If $q=0$, then ${ }^{m} A_{p-1}+k \equiv 0$, or $\equiv 1$, $(\bmod p)$, according as $m$ is, or is not, a multiple of $p$.

It is the purpose of the present paper to show that the Wilson-Glaisher theorem, the Moritz theorem, and other theorems are special cases of a still more general theorem relating to the symmetric functions of special systems of numbers, these systems being composed of the residues of powers, eventually repeated, for different moduli.

2. The Generalized Theorem. We shall prove the following general theorem.

Let $m=p^{\alpha} q^{\beta} \cdots r^{\gamma},(p, q, \cdots, r$ odd primes, $p<q<\cdots<r$; $\alpha \geqq 1, \beta \geqq 1, \cdots, \gamma \geqq 1)$, be an odd number and let $\rho, \sigma, \cdots, \chi$ be any divisors respectively of $\phi\left(p^{\alpha}\right), \phi\left(q^{\beta}\right), \cdots, \phi\left(r^{\gamma}\right)$, where $\phi(n)$ denotes Euler's Indicator; we shall write $\rho=p^{a} \lambda(\lambda$ divisor

* J. W. L. Glaisher, Congruences relating to the sums of products of the first $n$ numbers and to other sums of products, Quarterly Journal of Mathematics, vol. 31 (1900), pp. 1-35; see p. 23. See also L. E. Dickson, History of the Theory of Numbers, vol. I, p. 99.

$\dagger$ R. E. Moritz, On an extension of Glaisher's generalization of Wilson's theorem, Tôhoku Mathematical Journal, vol. 28 (1927), p. 198-201. 
of $p-1), \sigma=q^{b} \mu$ ( $\mu$ divisor of $\left.q-1\right), \cdots, \chi=r^{c} \nu$ ( $\nu$ divisor of $r-1)$. Let $\left(u_{0}, u_{1}, \cdots, u_{\rho-1}\right),\left(v_{0}, v_{1}, \cdots, v_{\rho-1}\right), \cdots,\left(w_{0}, w_{1}, \cdots\right.$, $\left.w_{\chi-1}\right)$ be the complete root systems respectively of the congruences

$$
u^{\rho} \equiv 1\left(\bmod p^{\alpha}\right) ; v^{\sigma} \equiv 1\left(\bmod q^{\beta}\right) ; \cdots ; w^{\chi} \equiv 1\left(\bmod r^{\gamma}\right) .
$$

Consider the $\tau=\rho \sigma \cdots \chi$ numbers

$$
t_{1}, t_{2}, \cdots, t_{r}
$$

two by two incongruent $(\bmod m)$, represented by the form

$$
\begin{gathered}
A u_{d}+B v_{e}+\cdots+C w_{f} \\
(0 \leqq d \leqq \rho-1,0 \leqq e \leqq \sigma-1, \cdots, 0 \leqq f \leqq \chi-1)
\end{gathered}
$$

in which $A, B, \cdots, C$ denote auxiliary integers satisfying the congruences

$$
\begin{aligned}
& A \equiv 1\left(\bmod p^{\alpha}\right) ; \quad B \equiv 1\left(\bmod q^{\beta}\right) ; \quad \cdots ; \quad C \equiv 1\left(\bmod r^{\gamma}\right) \\
& A \equiv 0\left(\bmod \frac{m}{p^{\alpha}}\right) ; \quad B \equiv 0\left(\bmod \frac{m}{q^{\beta}}\right) ; \quad \cdots ; \quad C \equiv 0\left(\bmod \frac{m}{r^{\gamma}}\right) .
\end{aligned}
$$

Consider the $k \tau$ integers $t_{j, i},(j=1,2, \cdots, \tau ; i=1,2, \cdots, k)$, $k$ by $k$ congruent $(\bmod m)$, precisely,

(1) $\quad t_{j, i} \equiv t_{j}(\bmod m), \quad(i=1,2, \cdots, k), \quad j=1,2, \cdots, \tau$, and $h$ other arbitrary integers

$$
z_{1}, z_{2}, \cdots, z_{h}, \quad(h \geqq 0) .
$$

If $R_{s \tau}(m ; \rho, \sigma, \cdots, \chi \| k, h)$ denotes the sum of the products of integers (1) and (2) taken $s \tau$ together, $\left(R_{0}=1\right)$, then from any one of the inequalities

$$
h<\lambda, h<\mu, \cdots, h<\nu
$$

there follows the corresponding congruence 


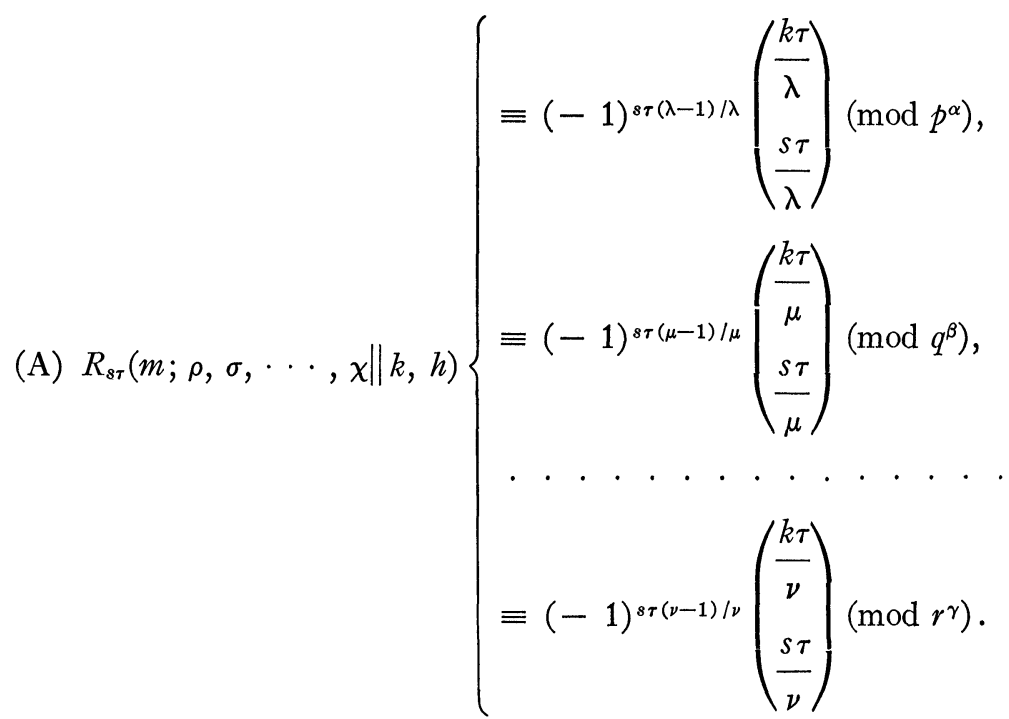

In the special case $s=k, h<\lambda, h<\mu, \cdots, h<\nu$, if the integers

$$
\frac{k \tau}{\lambda}(\lambda-1), \frac{k \tau}{\mu}(\mu-1), \cdots, \frac{k \tau}{\nu}(\nu-1)
$$

are all even or all odd, we have the congruence

$$
R_{k \tau}(m ; \rho, \sigma, \cdots, \chi \| k, h) \equiv \pm 1(\bmod m) .
$$

3. Special Cases. We observe that the $\phi(m)$ integers $(\bmod m)$, prime to $m$, are characterized by their satisfying the congruences $t^{\phi\left(p^{\alpha}\right)} \equiv 1\left(\bmod p^{\alpha}\right) ; \quad t^{\phi\left(q^{\beta}\right)} \equiv 1\left(\bmod q^{\beta}\right) ; \quad \cdots ; \quad t^{\phi\left(r^{\gamma}\right)} \equiv 1\left(\bmod r^{\gamma}\right)$. Therefore we may express Wilson's classic theorem and its generalizations by means of the forms

$$
\begin{array}{lll}
R_{p-1}(p ; p-1 \| 1,0) & \equiv-1(\bmod p) & (\text { WiLsoN })^{*}, \\
R_{\phi\left(p^{\alpha}\right)}\left(p^{\alpha} ; \phi\left(p^{\alpha}\right) \| 1,0\right) & \equiv-1\left(\bmod p^{\alpha}\right) \\
R_{\phi(m)}\left(m ; \phi\left(p^{\alpha}\right), \phi\left(q^{\beta}\right), \cdots, \phi\left(r^{\gamma}\right) \| 1,0\right) & \equiv 1(\bmod m) & \\
R_{p-1}(p ; p-1 \| k, h) & \equiv-k(\bmod p) & \text { GLAISHER } \dagger, \\
& \text { MORITZ } \ddagger
\end{array}
$$

* See L. E. Dickson, op. cit., p. 62.

$\dagger$ See L. E. Dickson, op. cit., p. 65.

† See L. E. Dickson, op. cit., p. 99; and R. E. Moritz, loc. cit. 


$$
\left.\begin{array}{c}
R_{s \tau}\left(m ; \phi\left(p^{\alpha}\right), \phi\left(q^{\beta}\right), \cdots, \phi\left(r^{\gamma}\right) \| k, 0\right) \\
\left(\tau=\phi(m)=\phi\left(p^{\alpha}\right) \cdots \phi\left(r^{\gamma}\right)\right) \\
(\mathrm{M} . \mathrm{BAUER})^{*}
\end{array}\right\} \equiv(-1)^{s \tau /(p-1)}\left(\begin{array}{c}
\frac{k \tau}{p-1} \\
\frac{s \tau}{p-1}
\end{array}\right)\left(\bmod p^{\alpha}\right),
$$

For the $\omega$-ic residues $\left(\bmod p^{\alpha}\right)$,

$$
R_{\rho}\left(p^{\alpha} ; \rho \| 1,0\right) \equiv(-1)^{\rho-1}\left(\bmod p^{\alpha}\right) ; \quad \rho=\frac{\phi\left(p^{\alpha}\right)}{D\left(\omega, \phi\left(p^{\alpha}\right)\right)} \dagger .
$$

And also

(3) $R_{\varepsilon \lambda}(m ; \rho, \sigma, \cdots, \chi \| 1,0) \equiv(-1)^{s(\lambda-1)}\left(\begin{array}{c}\frac{\tau}{\lambda} \\ s\end{array}\right),\left(\bmod p^{\alpha}\right), \quad(\mathrm{RICCI}) . \ddagger$

4. Proof. Let $R_{n_{i}}(m ; \rho, \sigma, \cdots, \chi \| 1,0)$ denote the sum of the products of the $\tau$ numbers $t_{j, i},(j=1,2, \cdots, \tau)$, of the system (1) taken $n_{i}$ together, and let $R_{n}^{\prime}$ be the sum of the products of the $h$ numbers $z_{1}, z_{2}, \cdots, z_{h}$ taken $n$ together $\left(R_{n}^{\prime}=1\right.$, if $\left.h n=0\right)$. Obviously we have the equality

(4) $R_{s \tau}(m ; \rho, \sigma, \cdots, \chi \| k, h)$

$$
=\sum\left\{\prod_{i=1}^{k} R_{n_{i}}(m ; \rho, \sigma, \cdots, \chi \| 1,0)\right\} R_{n}^{\prime},
$$

* See L. E. Dickson, op. cit., p. 88. (Bauer ${ }^{186}$.)

† See P. Bachmann, Niedere Zahlentheorie, Teil I, Leipzig, 1902, p. 347.

¥ See G. Ricci, Sulle funzioni simmetriche delle radici dell'unità secondo un modulo composto, Annali di Matematica, (4), vol. 9 (1931), p. 190, formula $\left(\mathrm{B}_{4}\right)$. 
in which the sum is extended to the solutions in integers $\geqq 0$ of the equation

$$
\begin{gathered}
n_{1}+n_{2}+\cdots+n_{k}+n=s \tau, \\
\left(0 \leqq n_{i} \leqq \tau, i=1,2, \cdots, k ; 0 \leqq n \leqq h\right) .
\end{gathered}
$$

If $n_{i} \not \equiv 0(\bmod \lambda)$, then it is known* that

$$
R_{n_{i}}(m ; \rho, \sigma, \cdots, \chi \| 1,0) \equiv 0\left(\bmod p^{\alpha}\right),
$$

and if $n_{i}=s \lambda$, then the congruence (3) stands. Therefore if one at least of the integers $n_{1}, n_{2}, \cdots, n_{k}$ is $\neq \equiv 0(\bmod \lambda)$, then the corresponding term on the right of (4) is divisible by $p^{\alpha}$; hence, for the relation $0 \leqq n \leqq h<\lambda$, we obtain

$$
\begin{aligned}
R_{s \tau}(m ; \rho, \sigma, \cdots, \chi \| k, h) & \\
\equiv & \sum \prod_{i=1}^{k} R_{s_{i} \lambda}(m ; \rho, \sigma, \cdots, \chi \| 1,0)\left(\bmod p^{\alpha}\right), \\
& \left(s_{1}+s_{2}+\cdots+s_{k}=s \frac{\tau}{\lambda} ; 0 \leqq s_{i} \leqq \frac{\tau}{\lambda}\right) .
\end{aligned}
$$

Then, by (3), we may write

$$
\begin{array}{r}
R_{s \tau}(m ; \rho, \sigma, \cdots, \chi \| k, h) \equiv(-1)^{s \tau(\lambda-1) / \lambda} \sum \prod_{i=1}^{k}\left(\begin{array}{c}
\frac{\tau}{\lambda} \\
s_{i}
\end{array}\right)\left(\bmod p^{\alpha}\right) \\
\left(s_{1}+s_{2}+\cdots+s_{k}=\frac{s \tau}{\lambda}\right) ;
\end{array}
$$

and, by a well known formula on binomial coefficients, from this congruence we deduce the first formula of (A). We may deduce the other formulas in a similar manner.

R. Scuola Normale Superiore

$$
\text { Pisa, Italy }
$$

* See G. Ricci, loc. cit., formula $\left(\mathrm{B}_{4}\right)$. 